\title{
Mucosal Expression of Type 2 and Type 17 Immune Response Genes Distinguishes Ulcerative Colitis From Colon-Only Crohn's Disease in Treatment-Naive Pediatric Patients
}

\author{
Michael J. Rosen, ${ }^{1,2}$ Rebekah Karns, ${ }^{1}$ Jefferson E. Vallance, ${ }^{1}$ Ramona Bezold, ${ }^{1}$ \\ Amanda Waddell, ${ }^{1}$ Margaret H. Collins, ${ }^{3,4}$ Yael Haberman, ${ }^{1,5}$ Phillip Minar, ${ }^{1,2}$ \\ Robert N. Baldassano, ${ }^{6}$ Jeffrey S. Hyams, ${ }^{7}$ Susan S. Baker, ${ }^{8}$ Richard Kellermayer, ${ }^{9}$ \\ Joshua D. Noe, ${ }^{10}$ Anne M. Griffiths, ${ }^{11}$ Joel R. Rosh, ${ }^{12}$ Wallace V. Crandall, ${ }^{13}$ \\ Melvin B. Heyman, ${ }^{14}$ David R. Mack, ${ }^{15}$ Michael D. Kappelman, ${ }^{16}$ James Markowitz, ${ }^{17}$ \\ Dedrick E. Moulton, ${ }^{18}$ Neal S. Leleiko, ${ }^{19}$ Thomas D. Walters, ${ }^{11}$ Subra Kugathasan, ${ }^{20}$ \\ Keith T. Wilson, ${ }^{21,22}$ Simon P. Hogan, ${ }^{2,23}$ and Lee A. Denson ${ }^{1,2}$
}

${ }^{1}$ Division of Gastroenterology Hepatology and Nutrition ${ }^{3}$ Division of Pathology ${ }^{23}$ Division of Allergy and Immunology, Cincinnati Children's Hospital Medical Center, Cincinnati, Ohio; 'Department of Pediatrics, "Department of Pathology' and Laboratory Medicine, University of Cincinnati College of Medicine, Cincinnati, Ohio; ${ }^{5}$ Pediatric Gastroenterology Unit, The Edmond and Lily Safra Children's Hospital, Sheba Medical Center, Tel Hashomer, Israel; ${ }^{6}$ Division of Pediatric Gastroenterology, Hepatology and Nutrition, Children's Hospital of Philadelphia, Philadelphia, Pennsylvania; ${ }^{7}$ Division of Digestive Diseases, Hepatology, and Nutrition, Connecticut Children's Medical Center, Hartford, Connecticut; ${ }^{8}$ Digestive Diseases and Nutrition Center, Women and Children's Hospital of Buffalo, Buffalo, New York; ${ }^{9}$ Department of Pediatrics, Section of Pediatric Gastroenterology, Hepatology and Nutrition, Baylor College of Medicine, Houston, Texas; ${ }^{10}$ Division of Gastroenterology, Hepatology and Nutrition, Department of Pediatrics, Medical College of Wisconsin, Milwaukee, Wisconsin;

${ }^{11}$ Division of Gastroenterology, Hepatology and Nutrition. The Hospital for Sick Children, Toronto, Canada; ${ }^{12}$ Gorqueb Children's HospitallAtlantic Hejalth, Icahn School of Medicine at Mount Sinai, New York, New York; 'Division of Pediatric Gastroenterology, Hepatology and Nutrition, Nationwide Children's Hospital, Columbus, Ohio; ${ }^{14}$ Division of Pediatric Gastroenterology, Hepatology and Nutrition, University of California San Francisco, San Francisco, California; ${ }^{15}$ Department of Pediatrics and Children's Hospital of Eastern Ontario Inflammatory Bowel Disease Centre, Children's Hospital of Eastern Ontario, Ottawa, Ontario, Canada; ${ }^{16}$ Division of Pediatric Gastroenterology, Department of Pediatrics, University of North Carolina at Chapel Hill, Chapel Hill, North Carolina; ${ }^{17}$ Division of Pediatric Gastroenterology and Nutrition, Cohen Children's Medical Center of New York, New Hyde Park, New York; ${ }^{18}$ Division of Gastroenterology, Hepatology, and Nutrition, Department of Pediatrics, Vanderbilt University School of Medicine, Nashville, Tennessee; ${ }^{19}$ Division of Pediatric Gastroenterology, Nutrition and Liver Diseases, Hasbro Children's Hospital, Providence, Rhode Island; ${ }^{20}$ Division of Gastroenterology, Hepatology and Nutrition, Department of Pediatrics, Emory University, Atlanta, Georgia; ${ }^{21}$ Division of Gastroenterology, Hepatology and Nutrition, Department of Medicine, Vanderbilt University Medical Center, Nashville, Tennessee; ${ }^{22}$ Veterans Affairs Tennessee Valley Healthcare System, Nashville, Tennessee

BACKGROUND \& AIMS: There is controversy regarding the role of the type 2 immune response in the pathogenesis of ulcerative colitis (UC) - few data are available from treatment-naive patients. We investigated whether genes associated with a type 2 immune response in the intestinal mucosa are up-regulated in treatment-naive pediatric patients with UC compared with patients with Crohn's disease (CD)-associated colitis or without inflammatory bowel disease (IBD), and whether expression levels are associated with clinical outcomes. METHODS: We used a real-time reverse-transcription quantitative polymerase chain reaction array to analyze messenger RNA (mRNA) expression patterns in rectal mucosal samples from 138 treatment-naive pediatric patients with IBD and macroscopic rectal disease, as well as those from 49 children without IBD (controls), enrolled in a multicenter prospective observational study from 2008 to 2012. Results were validated in real-time reverse-transcription quantitative polymerase chain reaction analyses of rectal RNA from an independent cohort of 34 pediatric patients with IBD and macroscopic rectal disease and 17 controls from Cincinnati Children's Hospital Medical Center. RESULTS: We measured significant increases in mRNAs associated with a type 2 immune response (interleukin [IL]5 gene, IL13, and IL13RA2) and a type 17 immune response (IL17A and IL23) in mucosal samples from patients with UC compared with patients with colon-only CD. In a regression model, increased expression of $I L 5$ and $I L 17 A$ mRNAs distinguished patients with UC from patients with colon-only $\mathrm{CD}(P=.001$; area under the receiver operating characteristic curve, 0.72). We identified a gene expression pattern in rectal tissues of patients with UC, characterized by detection of IL13 mRNA, that predicted clinical response to therapy after 6 months (odds ratio [OR], 6.469; 95\% confidence interval [CI], 1.553-26.94), clinical response after 12 months (OR, 6.125; 95\% CI, 1.330-28.22), and remission after 12 months (OR, 5.333; 95\% CI, 1.132-25.12). CONCLUSIONS: In an analysis of rectal tissues from treatment-naive pediatric patients with IBD, we observed activation of a type 2 immune response during the early course of UC. We were able to distinguish patients with UC from those with colon-only CD based on increased mucosal 


\section{EDITOR'S NOTES}

\section{BACKGROUND AND CONTEXT}

There is controversy over the role of the type 2 immune response in pathogenesis of ulcerative colitis (UC) - few data are available from treatment-naïve patients.

\section{NEW FINDINGS}

The researchers showed that treatment-naïve pediatric patients with ulcerative colitis exhibit increased mucosal expression of genes associated with type 2 and type 17 immune responses compared to those with colon-only Crohn's disease.

\section{LIMITATIONS}

This study measured only mRNA expression and not protein abundance.

\section{IMPACT}

Expression of type 2 and type 17 immune response genes distinguishes ulcerative colitis from colon-only Crohn's disease in treatment-naïve pediatric patients.

expression of genes that mediate type 2 and type 17 immune responses. Increased expression at diagnosis of genes that mediate a type 2 immune response is associated with response to therapy and remission in pediatric patients with UC.

Keywords: Immune Regulation; Gene Expression Profile; Prognostic Factor; AUROC.

$\mathrm{T}$ ype 2 inflammation has been implicated in the pathogenesis of ulcerative colitis (UC) ${ }^{1,2}$ Classic type 2 immune responses are defined by production of the cytokines interleukin (IL)4, IL5, and IL13 by T helper cells and innate lymphoid cells and are involved in the expulsion of helminths and the pathogenesis of allergic diseases. ${ }^{3} \mathrm{UC}$, however, has been associated with an atypical type $2 \mathrm{im}-$ mune response, with increased IL5 and IL13, but not IL4. ${ }^{2}$ Further studies have indicated pathogenic effects of IL13 including activation of inflammatory colon mucosal natural killer $\mathrm{T}$ cells and impairment of epithelial barrier function. ${ }^{4}$ The initial observation of this atypical type 2 immune response in UC originated from ex vivo experiments with lamina propria immune cells isolated from surgical specimens from adults with IBD. ${ }^{2}$ Whether type 2 inflammation is involved in UC patients at diagnosis, before treatments that affect the inflammatory response, or in pediatric UC remains unknown. Furthermore, it is unknown if patients with heightened type 2 inflammatory responses attain different clinical outcomes.

Distinguishing UC from Crohn's colitis can be a diagnostic challenge in pediatric patients. Regardless of diagnosis with UC or Crohn's disease (CD), a colitis phenotype is a common feature of pediatric IBD. UC and inflammatory bowel disease (IBD) unclassified account for $30 \%-40 \%$ of pediatric IBD in the United States and Europe ${ }^{5-7}$ Furthermore, approximately $80 \%$ of children with $\mathrm{CD}$ have colonic involvement, with $25 \%$ showing a colon-only phenotype with no small intestinal involvement. ${ }^{8,9}$ Colon-only CD phenotype is even more common with younger age, occurring in approximately $40 \%$ of children younger than 10 years of age. ${ }^{8,9}$ Taken together, approximately $50 \%$ of pediatric IBD patients show an isolated colitis phenotype. Many of these children show overlapping or atypical features, which hinder rendering a specific diagnosis of $C D$ or UC. ${ }^{10}$ It is not known whether mucosal expression of genes associated with type 2 inflammation can distinguish UC and CD pediatric patients with isolated colitis phenotypes.

We hypothesized that treatment-naive pediatric patients with UC would show increased mucosal type 2 immune responses compared with patients without IBD and Crohn's colitis, and that high expression of type 2-associated genes would be associated with poor response to therapy in pediatric UC. Here, we applied a microfluidic real-time reverse-transcription quantitative polymerase chain reaction (RT-qPCR) array platform to determine rectal mucosal expression of genes associated with type 1, type 2 , and type 17 inflammation in patients with UC, Crohn's colitis, and non-IBD controls from a large multicenter North American pediatric IBD inception cohort. We report that expression of genes associated with type 2 and type 17 immune responses distinguished 2 colon-only phenotypes of pediatric IBD: UC and colon-only CD. Furthermore, we observed that a gene expression profile marked by detectable IL13 expression is associated with improved clinical outcomes in pediatric UC.

\section{Materials and Methods}

\section{RISK Cohort Rectal RNA Samples}

Rectal mucosal RNA samples from treatment-naive IBD patients and non-IBD controls and associated clinical data were obtained from the Risk Stratification and Identification of Immunogenetic and Microbial Markers of Rapid Disease Progression in Children with Crohn's Disease (RISK) study, a prospective observational IBD inception cohort sponsored by the Crohn's and Colitis Foundation. A total of 1812 children and adolescents younger than age 17, newly diagnosed with IBD and non-IBD controls, were enrolled at 28 North American pediatric gastroenterology centers between 2008 and 2012. All participants underwent baseline colonoscopy with confirmation of characteristic chronic active colitis and/or ileitis by histology before diagnosis and treatment. Institutional Review Board approval was obtained locally at each participating site. All endoscopic tissues obtained in the RISK study were stored in RNALater (Thermo Fisher Scientific, Waltham, MA), thus

Abbreviations used in this paper: CD, Crohn's disease; CDc, colon-only Crohn's disease; CDic, ileocolonic Crohn's disease; $\mathrm{Cl}$, confidence interval; Cq, quantification cycle; IBD, inflammatory bowel disease; ICOS, inducible t-cell costimulator; IFNG, interferon- $\gamma$; IL, interleukin; OR, odds ratio; PGA, physician's global assessment; RHI, Robarts Histopathology Index; RISK, Risk Stratification and Identification of Immunogenetic and Microbial Markers of Rapid Disease Progression in Children with Crohn's Disease; RT-qPCR, quantitative reverse-transcription polymerase chain reaction; TNF, tumor necrosis factor; UC, ulcerative colitis. 
RNA and DNA, but not protein, are available for study. This analysis included a representative subgroup of RISK participants with UC $(n=56)$, colon-only CD (CDc, $n=36)$, ileocolonic CD (CDic, $n=46$ ), and non-IBD controls $(n=49)$. This constitutes all participants within RISK with rectal RNA who met study criteria for UC and CDc, and a random sample of those who met study criteria for CDic. Only patients with a confirmed diagnosis of CD, UC, or non-IBD based on standard clinical and pathologic criteria after a median of 3.3 years of follow-up evaluation were included in this analysis. To meet the study definition of CD in RISK, patients eventually must have been found to have at least 2 of the following: signs or symptoms consistent with CD (diarrhea, abdominal pain, rectal bleeding, malaise, weight loss, or linear growth failure), endoscopic findings of discontinuous ulceration or cobblestoning, and/or histopathologic findings of patchy inflammatory cell infiltrates or epithelial granuloma. Detailed granular data regarding anatomic disease involvement was obtained for all participants in the RISK cohort. For the purposes of this analysis, all participants with UC and CD must have shown macroscopic inflammation in the rectum at the time of biopsy collection. Participants with UC must have received a most recent diagnosis of UC, shown macroscopic inflammation in the rectum (because RNA analyzed in this study was isolated from rectal biopsy specimens), absence of macroscopic inflammation in the ileum at their enrollment endoscopy, and absence of evidence of jejunal inflammation. Participants with CDc must have received a most recent diagnosis of CD and shown macroscopic inflammation in the rectum with an absence of macroscopic inflammation in the ileum or jejunum. Participants with CDic must have received a most recent diagnosis of $\mathrm{CD}$ and shown macroscopic inflammation in both the rectum and ileum. Non-IBD control participants must have received a most recent diagnosis of non-IBD and shown macroscopically and microscopically normal ileum and colon.

\section{Cincinnati Cohort}

Under a protocol approved by the Cincinnati Children's Hospital Medical Center Institutional Review Board, patients presenting for routine colonoscopy for clinical indications were enrolled in a separate independent local Cincinnati cohort for the purposes of validating the findings from the RISK cohort. Patients in the Cincinnati cohort were not in the RISK cohort. Clinical information was collected at the time of enrollment. Rectal biopsy specimens were placed in RNALater and stored at $-80^{\circ} \mathrm{C}$. Only patients with macroscopic inflammation in the rectum at endoscopy were included in the analysis. H\&Estained sections from rectal biopsy specimens were scored by a pediatric pathologist blinded to diagnosis using the validated Robarts Histopathology Index (RHI). ${ }^{11}$

\section{Real-Time RT-qPCR}

For all RISK cohort RNA samples studied, rectal RNA integrity was determined using an Agilent 2100 Bioanalyzer instrument (Agilent Technologies, Santa Clara, CA). Only samples with an RNA integrity number of 7 or greater were included ( $95.5 \%$ of samples tested). The expression of 24 genes related to type 2 , type 1 , type 17 , and regulatory immune responses (Supplementary Table 1) was determined by quantitative real-time RT-qPCR in duplicate from $100 \mathrm{ng}$ starting RNA using custom TaqMan array 384-well microfluidic cards on a 7900HT Real-Time PCR System (Thermo Fisher Scientific). The endogenous reference control was selected empirically by analyzing a subset of 16 samples (4 from each diagnosis group) with the TaqMan Human Endogenous Control Array (Thermo Fisher Scientific), which assesses expression of 16 genes known to show minimal differential expression across tissues. Real-time RT-qPCR on rectal RNA from the Cincinnati cohort was performed using a subset of individual gene expression assays from the custom microfluidic array. Relative expression was determined using a modification of the $2^{-\Delta \Delta \mathrm{Cq}}$ method as previously described. ${ }^{12}$ Briefly, changes in the quantification cycle $(\Delta \mathrm{Cq})$ values were obtained by subtracting the target $\mathrm{Cq}$ from that of the reference gene. $\Delta \mathrm{Cq}$ values then were shifted such that expression in samples with undetectable expression $(\mathrm{Cq}>40)$ was considered half that of the sample with the least detectable expression.

\section{Outcomes}

Outcomes assessed in the UC group included steroid-free, surgery-free clinical response and clinical remission at 6 and 12 months. Clinical remission was defined as a physician global assessment (PGA) of inactive disease. The 4 PGA categories were inactive mild, moderate, and severe disease. Clinical response was defined as a PGA of inactive or mild disease, with at least a 1-category decrease in PGA between baseline and the indicated time point (ie, if the patient had mild disease at baseline, they must have improved to inactive disease). For both end points, patients must have been off systemic corticosteroids, with no prior surgery at the indicated time point.

\section{Statistical Analysis}

Statistical analyses were performed using SAS v9.3 (SAS Institute, Cary, NC). For gene expression array data from the RISK cohort, we assessed global differences in expression for each gene among the 4 diagnosis groups using the nonparametric Kruskal-Wallis $\mathrm{H}$ test with false-discovery rate to control the type 1 error rate at 0.05 . We then performed pairwise comparisons between diagnosis groups only for genes passing the omnibus test using the Mann-Whitney $U$ test with false-discovery rate correction. Correlation of expression between genes was assessed by the Spearman rank correlation coefficient. Principal component analysis incorporating all array gene expression data was performed using Genespring GS (Agilent Technologies). We applied unsupervised hierarchical clustering (Genespring GS) to identify patient clusters with unique gene expression patterns. To validate gene expression differences discovered in the RISK cohort in the independent Cincinnati cohort, we compared expression of selected genes and RHI using the Kruskal-Wallis $\mathrm{H}$ test followed by pairwise comparisons using the Mann-Whitney $U$ test only if the omnibus test was significant. In a sensitivity analysis to control for histologic disease activity within the local Cincinnati cohort, patients with CD and UC were matched on RHI (within 1 point) and gene expression was compared using the Wilcoxon signed-rank test. We assessed the performance of gene expression to distinguish UC from $\mathrm{CDc}$ and to predict clinical outcomes in UC patients using logistic regression. The regression model for distinguishing UC from CDc was validated internally across 1000 random samples of equal size 
using a random sampling with replacement bootstrap technique. To assess the contribution of degree of inflammation to the observed differences in gene expression between UC and $\mathrm{CDc}$, bivariate logistic regression was performed for each target gene with significant differences in gene expression between UC and CDc and S100A8. A change in effect estimate between univariate and bivariate analysis with $S 100 A 8$ of less than $10 \%$, $10 \%-20 \%$, or greater than $20 \%$ were interpreted as no, mild, or moderate confounding by $100 A 8$, respectively. ${ }^{13}$ S100A8 was forced into the final multivariate model for predicting UC from $\mathrm{CDc}$ to determine the contribution of the degree of inflammation to the final model. Baseline characteristics among gene expression patient clusters were compared using the Kruskal-Wallis $\mathrm{H}$ test for continuous variables or the chi-square test for nominal variables. The association between gene expression cluster and clinical outcomes in the UC group was assessed by the Fisher exact test.

\section{Sample Size and Power}

We determined that a sample size of 40 patients per diagnosis group would provide $90 \%$ power to detect a 1.5 -fold difference in expression while controlling the type I error rate at $1 \%$ for multiplicity.

\section{Results}

\section{RISK Cohort Rectal Mucosal Gene Expression}

Demographics and baseline characteristics of the subset of the RISK cohort participants studied for this analysis are detailed in Table 1 . There were no meaningful differences between the subset of RISK cohort patients included in this study and the overall RISK cohort other than for characteristics within the specific inclusion or exclusion criteria for this study (Supplementary Table 2). All participants with CDic, CDc, and UC in the studied subset showed macroscopic rectal inflammation at endoscopy, compared with $67.5 \%, 74.8 \%$, and $88.5 \%$, respectively, in the overall RISK cohort (relative rectal sparing can be an atypical feature of pediatric UC). We only included participants showing macroscopic rectal inflammation to limit the effect of differences in rectal inflammation on the analysis. In addition, no patients in the studied subset of CDc participants showed jejunal inflammation compared with $9.5 \%$ of CDc participants in the overall RISK cohort. Although jejunal involvement (Paris L4b) does not preclude the designation of colon-only CD (Paris L2) based on the Paris classification, ${ }^{14}$ we excluded participants with jejunal disease from our CDc group because these patients can be distinguished easily from UC patients based on anatomic disease distribution alone.

Among 16 candidate reference gene controls assessed, glyceraldehyde-3-phosphate dehydrogenase showed the least variable expression across diagnosis groups (SD of $\mathrm{Cq}, 0.55)$, and was included in the gene expression array as the endogenous control against which we normalized results (Supplementary Figure 1).

The rectal relative expression of the 22 genes assayed normalized to the median of non-IBD patients is detailed in Figure $1 A$ (the assay for CLDN2 did not amplify and is excluded from the results). Compared with non-IBD

Table 1.Baseline Characteristics of RISK Cohort Patients Studied

\begin{tabular}{|c|c|c|c|c|}
\hline & Non-IBD $(n=49)$ & CDic $(n=46)$ & CDc $(n=36)$ & $\cup C(n=56)$ \\
\hline Age, $y$ & $12.8(10.8,15)$ & $12.4(10.9,13.6)$ & $12.7(10.8,14.5)$ & $13.5(10.8,15.5)$ \\
\hline A1a: $0-<10$ y & & $11(23.9)$ & $6(17.1)$ & $13(23.2)$ \\
\hline A1b: $10-<17$ y & & $35(76.1)$ & $30(85.7)$ & $43(76.8)$ \\
\hline Male sex & $23(46.9)$ & $25(54.3)$ & $17(47.2)$ & $30(53.6)$ \\
\hline \multicolumn{5}{|l|}{ CD location } \\
\hline L1: terminal ileal \pm limited cecal disease & & $0(0)$ & $0(0)$ & \\
\hline L2: colonic & & $0(0)$ & $36(100)$ & \\
\hline L3: ileocolonic & & $46(100)$ & $0(0)$ & \\
\hline L4a: upper disease proximal to ligament of Treitz & & $32(69.6)$ & $17(47.2)$ & \\
\hline L4b: upper disease distal to ligament of Treitz & & $12(26.1)$ & $0(0)$ & \\
\hline \multicolumn{5}{|l|}{ UC extent } \\
\hline E1: ulcerative proctitis & & & & $3(5.3)$ \\
\hline E2: left-sided colitis & & & & 7 (12.5) \\
\hline E3: extensive colitis & & & & $9(16.1)$ \\
\hline E4: pancolitis & & & & $35(62.5)$ \\
\hline Data not available & & & & $2(3.6)$ \\
\hline Macroscopic rectal involvement & & $46(100)$ & $36(100)$ & $56(100)$ \\
\hline \multicolumn{5}{|l|}{ PGA } \\
\hline Inactive & & $0(0)$ & $1(2.8)$ & $1(1.8)$ \\
\hline Mild & & $11(23.9)$ & 7 (19.4) & $20(35.7)$ \\
\hline Moderate & & $22(47.8)$ & $19(52.8)$ & $23(41.1)$ \\
\hline Severe & & $13(28.3)$ & $9(25.0)$ & $12(21.4)$ \\
\hline PUCAI & & & & $45(35,60)$ \\
\hline Rectal deep ulcers & & $5(10.9)$ & $12(33.3)$ & $10(17.9)$ \\
\hline
\end{tabular}


patients, UC patients were the only group that showed significantly increased rectal expression of the genes for the type 2 cytokines IL5 and IL13 (no group showed significantly increased expression of IL4). IL17A and IL23A were increased significantly in UC and $\mathrm{CDc}$, but not in CDic, compared with non-IBD. Interferon- $\gamma$ and IL22 expression were increased similarly in all 3 groups compared with non-IBD.

Compared with CDc patients with macroscopic rectal involvement, UC patients showed significantly increased rectal expression of genes associated with type 2 ([IL5, IL13, IL13RA2, inducible t-cell costimulator [ICOS], and the transcript for membrane-bound IL33 receptor, IL1RL1[m]) and type 17 (IL17A, IL23A) immune responses. Of note, expression of the inflammatory marker S100A8, which encodes a subunit of calprotectin, was not significantly different between UC and CDc patients.

Consistent with the finding of increased IL13 and IL17A expression in UC patients, we observed moderate correlation $\left(\mathrm{r}^{2}=.18 ; P<.0001\right)$ between IL13 and IL17A expression among all IBD patients. However, among UC patients, we observed no significant correlation between IL13 and IL17A expression $\left(\mathrm{r}^{2}=.057 ; P=.076\right)$.

Principal component analysis (Figure $1 B$ ), incorporating all genes analyzed, identified 2 groups of patients. Group 1 included most of the UC and CDc patients, and group 2 included most of the non-IBD patients. CDic patients were intermixed between the 2 principal component groups.

Unsupervised hierarchical clustering segregated patients into 5 clusters based on gene expression (Figure $1 C$ ). The largest aggregate of a single diagnosis was UC patients within cluster 2 , which was defined by high expression of IL13 and IL5, and also high expression of CCL11, IL13RA2, CHI3L1, S100A8, IL23A, and IL17A. Cluster 2 was composed of $59.1 \%$ UC patients ( $46.4 \%$ of all the UC patients) and $6.8 \%$ non-IBD patients. The remaining clusters were defined by differences in IL5 and IL13 expression, with more variable and lower expression of the remaining genes compared with cluster 2. Cluster 4, which showed undetectable expression of IL5 and IL13, comprised the largest group of non-IBD patients $(43.5 \%$ non-IBD, $40.8 \%$ of all the non-IBD patients). This unequal distribution of diagnoses among the clusters was statistically significant, whereas other baseline characteristics were similar (Table 2).

\section{Validation of RISK Cohort Differential Rectal Gene Expression in the Cincinnati Cohort}

Demographics and baseline characteristics of patients in the Cincinnati cohort are detailed in Supplementary Table 3. Rectal biopsy specimens were analyzed from 17 non-IBD, $20 \mathrm{CD}$, and 14 UC patients. All CD and UC patients showed macroscopic inflammation in the rectum. Similar to the RISK Cohort, UC patients in our Cincinnati cohort showed increased rectal expression of IL5, IL13, IL13RA2, IL17A, and IL23A compared with CD patients with colitis and non-IBD patients (Figure 2A). Also consistent with the RISK cohort findings, UC and CD patients showed similarly increased IFNG expression compared with non-IBD patients.
We sought to compare rectal histopathologic disease activity between the macroscopically involved rectums of CD and UC patients in the Cincinnati cohort, acknowledging the challenge that no histopathologic index exists that has been validated for pediatric UC or $\mathrm{CD}$, or for comparing between CD and UC patients. Therefore, histopathologic activity was compared between patient groups using the RHI, a validated UC index that assesses the following features common to both UC and CD: chronic inflammatory infiltrate, lamina propria neutrophils, neutrophils in the epithelium, and erosion or ulceration. ${ }^{11}$ The median RHI was significantly higher in the macroscopically inflamed rectums of UC compared with CD patients (Supplementary Figure $2 A$ ). Therefore, to determine whether differences in gene expression are explained by differences in histopathologic severity, we performed a sensitivity analysis comparing gene expression between a subset of $10 \mathrm{UC}$ and CD patients matched on RHI (Supplementary Figure $2 B$ and $C$ ). In this smaller subset of RHI-matched patients, there was significantly increased rectal relative expression of IL13 and IL13RA2 in UC compared with CD patients. Furthermore, other gene expression relationships between UC and CD were maintained with numerically (but not statistically significantly) increased relative expression of IL17A, IL23A, and $I L 5$, and equivalent IFNG expression in UC compared with CD.

To determine if healing on treatment is associated with changes in IL13 and IL17A expression, we compared mucosal gene expression between UC patients in the Cincinnati cohort, all with active endoscopic disease, with 3 additional UC patients with complete mucosal healing on treatment (Mayo endoscopic score, 0; all female; ages 7, 9, and 20 years; healing achieved on 6-mercaptopurine + infliximab, mesalamine alone, and mesalamine and oral corticosteroids, respectively). Both IL13A and IL17A expression were decreased significantly in the patients with mucosal healing compared with those with active endoscopic disease (Figure 2B).

\section{IL5 and IL17 Expression Distinguish UC From Colitis-Only $C D$}

To determine the ability of gene expression to discriminate UC from CDc, we applied univariate logistic regression using only the genes with significant differential expression between UC and CDc (Supplementary Table 4). Using multivariate logistic regression, we determined that a model including IL5 and IL17A gene expression best balanced parsimony with discriminatory ability $(P=.001$; area under the curve, 0.72) (Table 3 and Figure $3 A$ ). We internally validated the model using bootstrap random sampling with replacement and showed that the bootstrapped estimates for the odds ratios (ORs) for both IL5 and IL17A were significant with $95 \%$ confidence intervals (CIs) not crossing 1 (Figure 3B).

Although there was not a significant difference in S100A8 expression between UC and CDc, we sought to determine the degree to which differences in other genes were confounded by general inflammatory activity. For 
genes that showed significantly different expression between UC and CDc, we compared the effect estimate obtained by univariate analysis with that obtained by bivariate analysis with $S 100 A 8$ (Supplementary Table 5). We detected no evidence of confounding by $S 100 A 8(<10 \%$ change in effect estimate) for $I L 5, I L 1 R L 1(m), I L 17 A$, and IL23A,
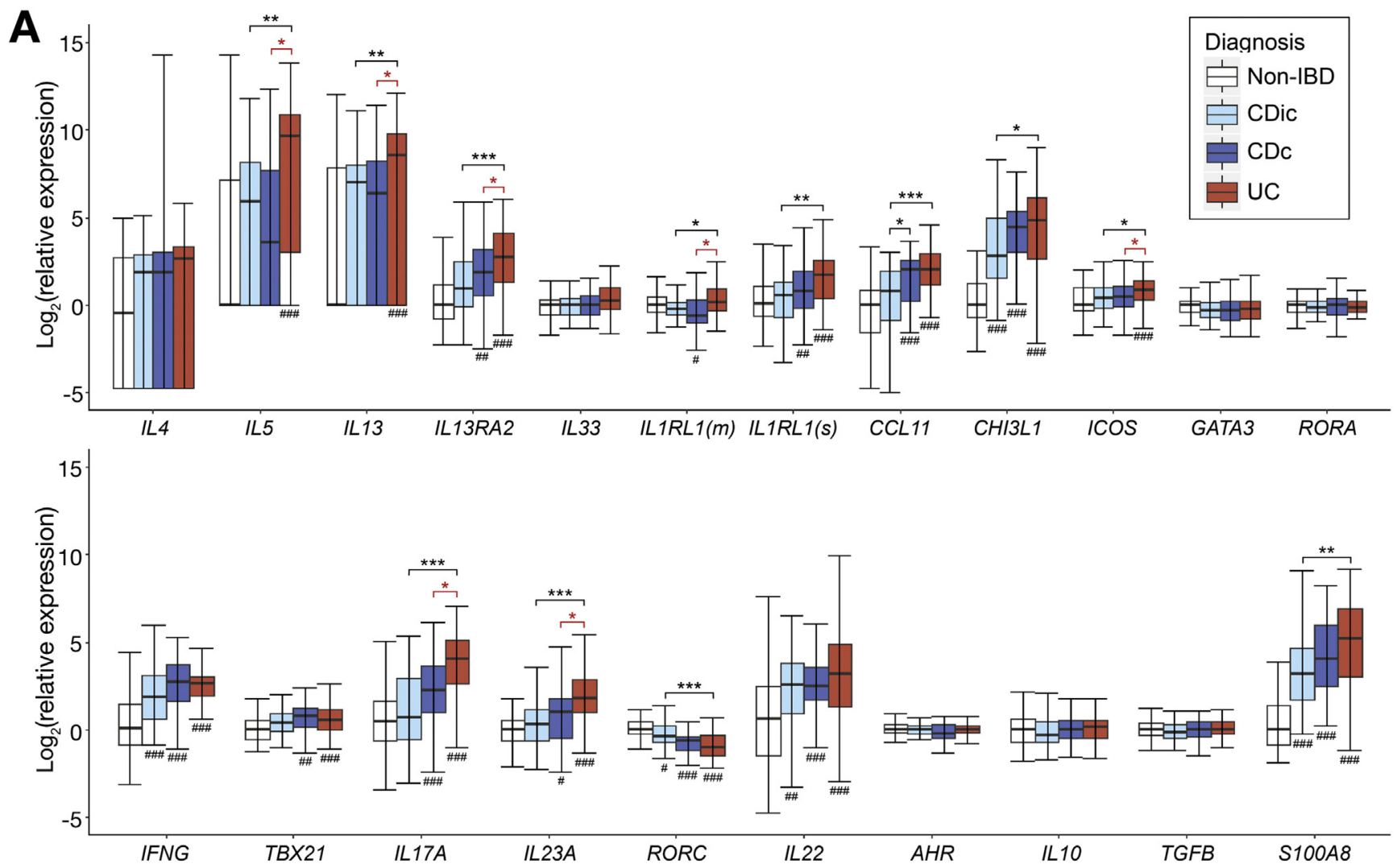

B

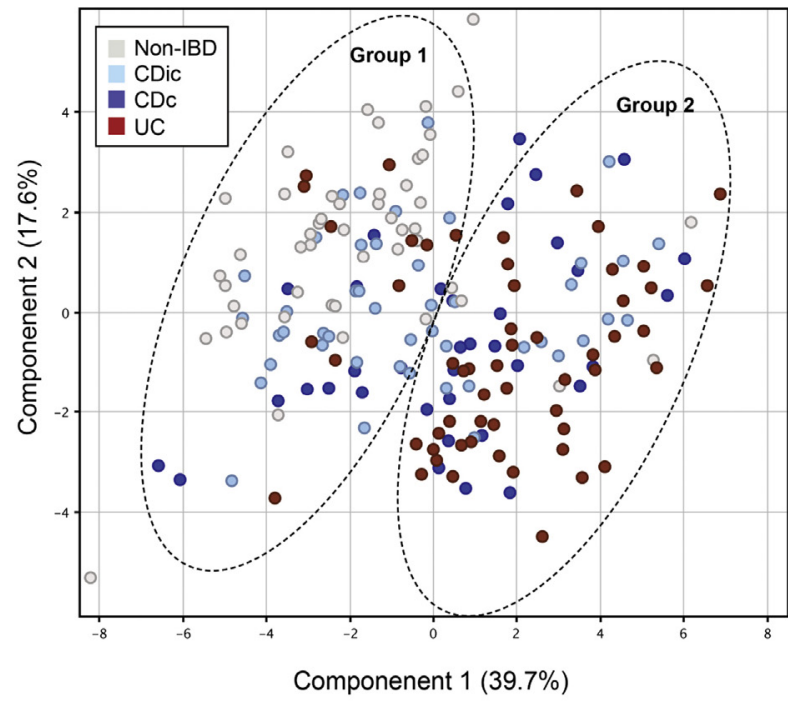

C

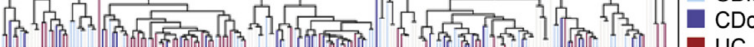
Condition:

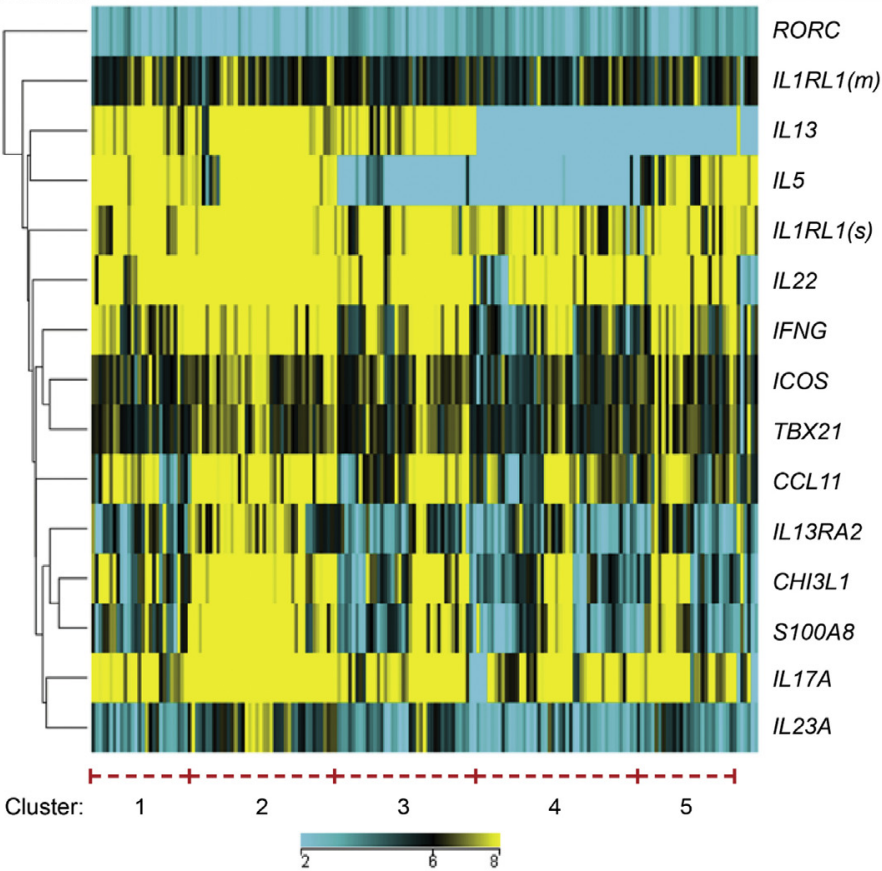


Table 2.Comparison of Baseline Characteristics Between Gene Expression Patient Clusters

Gene expression cluster

\begin{tabular}{|c|c|c|c|c|c|c|}
\hline & $1(n=25)$ & $2(n=44)$ & $3(n=39)$ & $4(n=46)$ & $5(n=27)$ & $P$ value \\
\hline Male sex & $11(44.0)$ & $23(52.3)$ & $25(64.1)$ & $28(60.9)$ & $12(44.4)$ & .342 \\
\hline Non-IBD & $6(24.0)$ & $3(6.8)$ & $9(23.1)$ & $20(43.5)$ & 7 (25.9) & \multirow[t]{4}{*}{$<.001$} \\
\hline CDic & $8(32.0)$ & $9(20.5)$ & $11(28.2)$ & $10(21.7)$ & 8 (29.6) & \\
\hline $\mathrm{CDC}$ & $4(16.0)$ & 6 (13.6) & $12(30.8)$ & 8 (17.4) & $6(22.2)$ & \\
\hline UC & 7 (28.0) & $26(59.1)$ & 7 (17.9) & 8 (17.4) & $6(22.2)$ & \\
\hline Inactive & $0(0)$ & $1(2.4)$ & $0(0)$ & $1(3.8)$ & $0(0)$ & \multirow[t]{4}{*}{$.119^{a}$} \\
\hline Mild & $3(15.8)$ & $17(41.5)$ & 7 (23.3) & $6(23.1)$ & $5(25.0)$ & \\
\hline Moderate & $14(73.7)$ & $13(31.7)$ & $15(50.0)$ & $10(38.5)$ & $10(50.0)$ & \\
\hline Severe & $2(10.5)$ & $10(24.4)$ & $8(26.7)$ & 9 (34.6) & $5(25.0)$ & \\
\hline Rectal deep ulcers & $4(21.1)$ & $7(17.1)$ & $6(20.0)$ & $6(23.1)$ & $3(15.0)$ & .957 \\
\hline
\end{tabular}

NOTE. Quantitative variables are expressed as medians (quartile 1, quartile 3), and dichotomous variables are shown as $\mathrm{n}(\%)$. Bold text indicates $P<.05$.

${ }^{a}$ Inactive and mild combined for chi-square test.

evidence of only mild confounding (10\%-20\% change) for IL13, and evidence of moderate confounding (20\%-30\%) for IL13RA2 and ICOS. Furthermore, when S100A8 is forced into our final model with $I L 5$ and $I L 17 A, S 100 A 8$ does not contribute any predictive value for UC over CDc to the model, and the overall model characteristics and performance are unchanged with an unchanged area under the curve of 0.72 (Supplementary Table 6).

\section{Gene Expression Predicts Clinical Outcome in UC Patients}

Of the 56 UC patients in the RISK cohort we studied, outcome data were available for 44 and 37 patients at 6 and 12 months, respectively. We applied univariate logistic regression to determine whether any of the 6 genes with differential expression between UC and CDc predicted steroid-free clinical remission or response at 6 or 12 months (Supplementary Table 7). We found that higher IL13 expression was associated significantly with an increased likelihood of clinical response at 6 (OR, 1.182; 95\% CI, 1.028-1.359) or 12 months (OR, 1.172; 95\% CI, 1.012-1.359), and a trend toward association with clinical remission at 12 months (OR, 1.126; 95\% CI, 0.978-1.297). We then assessed whether the unsupervised clustering based on gene expression predicted clinical outcomes in UC patients. We observed that patients in clusters 1,2 , and 3 were significantly more likely to show clinical response at 6 months (trend for remission), and clinical response and remission at 12 months, compared with those in clusters 4 and 5 (Table 4). The major distinguishing gene expression difference between these groups was increased IL13 gene expression in clusters 1-3 with essentially undetectable IL13 expression in clusters 4 and 5 . Baseline characteristics and medication exposures by 6 and 12 months were similar between UC patients in clusters 1-3 compared with clusters 3 and 4 , with the exception of exposure to anti-tumor necrosis factor (TNF) biologics, which occurred numerically, but not statistically significantly, more often in clusters 4 and 5 (Supplementary Table 8). By 6 months, $16.1 \%$ of patients in clusters $1-3$ and $30.8 \%$ of patients in clusters 4 and 5 were exposed to an anti-TNF biologic drug $(P=.414)$, and $25.0 \%$ of patients in clusters $1-3$ and $54.5 \%$ of patients in clusters 4 and 5 by 12 months $(P=.131)$. It is likely that the numerically increased and earlier infliximab exposure in clusters 4 and 5 is an additional reflection of the poorer clinical response of this group to first-line therapies (ie, corticosteroids, mesalamine, and thiopurines) compared with that of patients in clusters 1-3. We did not find any association between IL13 expression or gene expression cluster and clinical outcomes in CD.

Figure 1. Results of microfluidic RT-qPCR gene expression array on rectal mucosal RNA from RISK cohort patients. $(A)$ Box and whisker chart showing gene expression for each target gene on the array for each IBD diagnostic subphenotype normalized to median expression of the non-IBD patient group (boxes represent medians and interquartile range, whiskers represent the $95 \% \mathrm{Cl}$ ). (B) Principal component analysis plot showing separation of 2 groups of patients with UC and CDc clustering in group 1 and non-IBD in group 2. (C) Dendrogram and heatmap showing the results of unsupervised hierarchical clustering using genes with differential expression between at least 2 diagnosis groups. UC patients aggregate within cluster 2 , which shows high expression of $I L 13, I L 5$, and $I L 17 A$. IL1RL1(m) and ILRL1(s), transcripts for the membrane-bound and soluble versions of the IL33 receptor, respectively. ${ }^{\star} P<.05$, ${ }^{* \star} P<.01$, ${ }^{\star \star \star} P<.001$; ${ }^{\#} P<.05,{ }^{\# \#} P<.01$, and ${ }^{\# \# \#} P<.001$ vs nonIBD (all $P$ values are false-discovery rate-corrected). 


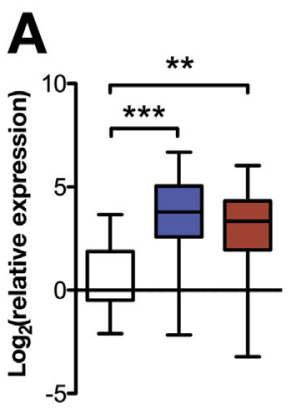

IFNG

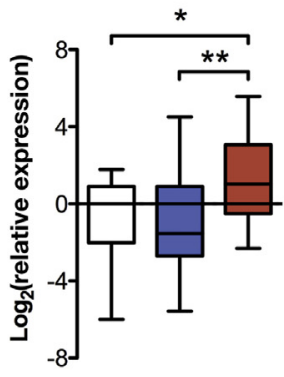

IL5

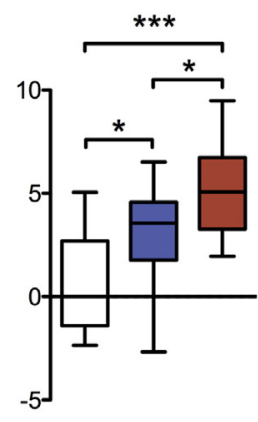

IL13

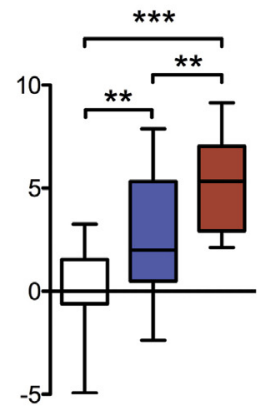

IL17A

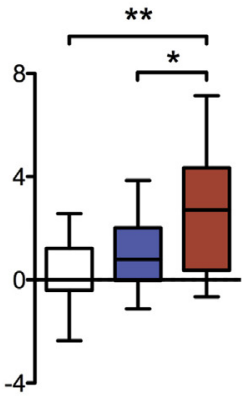

IL13RA2

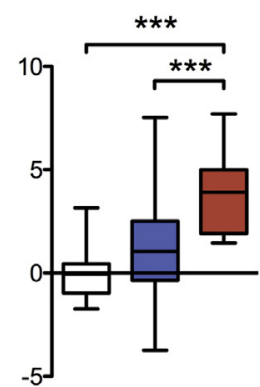

IL23A

B

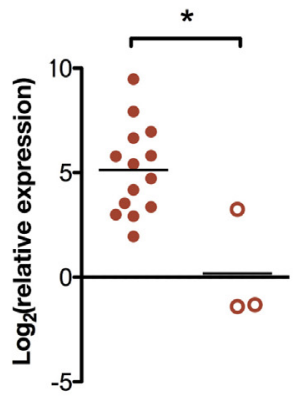

IL13

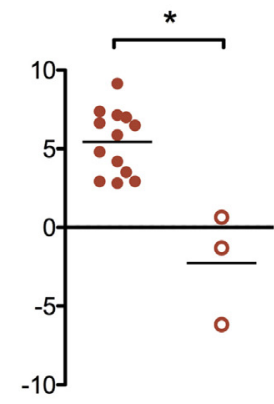

IL17A

Active UC

O Healed UC

Figure 2. Real-time RT-qPCR of rectal mucosal RNA from patients in the Cincinnati validation cohort. $(A)$ Box and whisker chart showing gene expression normalized to median expression of the non-IBD patient group (boxes represent medians and interquartile range, whiskers represent the $95 \% \mathrm{Cl}$ ). (B) Dot plot showing gene expression (normalized to median expression of the non-IBD patient group) in patients with active UC compared with UC patients with endoscopic healing (each dot represents a single patient, and lines represent the median). ${ }^{\star} P<.05,{ }^{\star \star} P<.01,{ }^{\star \star \star} P<.001$.

\section{Discussion}

In a well-characterized, treatment-naive, pediatric IBD inception cohort, we show that the rectal mucosa of pediatric UC patients was distinguished from that of patients with colon-only Crohn's disease by increased expression of genes associated with type 2 and type 17 immune responses. This finding was not explained by differences in overall inflammation as measured by $100 A 8$ expression. Furthermore, in an analysis of prospective data from this cohort, we show that heightened rectal mucosal IL13 expression at baseline is associated with improved clinical outcomes in pediatric UC.

The involvement of type 2 inflammation in the pathogenesis of UC has been debated in the literature. Fuss et $\mathrm{al}^{1}$ first described disparate cytokine secretion from lamina propria mononuclear cells isolated from surgical specimens of adults with UC and CD, with those from UC patients

Table 3. Multivariate Logistic Regression for Discriminating UC From CDc

$\begin{array}{lccc}\text { Gene } & \text { OR }^{a} & 95 \% \mathrm{Cl} & P \text { value } \\ \text { IL5 } & 1.130 & 1.032-1.238 & .009 \\ \text { IL17A } & 1.196 & 0.976-1.467 & .085\end{array}$

${ }^{a}$ Odds of a diagnosis of UC over CDc per unit increase in $\mathrm{Cq}$ value for the listed gene. producing increased IL5 and IL13 and those from CD patients producing interferon- $\gamma .^{1,2}$ The same group went on to show that IL13 is produced by natural killer T cells, and that both IL13 and natural killer T cells disrupt epithelial barrier function. $^{2,4}$ Accordingly, we previously showed increased epithelial activation of signal transducer and activator of transcription 6, a transcription factor downstream of IL13 signaling, in pediatric UC. ${ }^{15}$ However, other groups have not detected increased IL13 production either from lamina propria mononuclear cells or colon tissue of adult or pediatric patients, respectively, with UC, perhaps because of differences in the ex vivo experimental techniques for studies. ${ }^{16,17}$ Here, we provide strong evidence from a large well-characterized cohort that mucosal type 2 immune responses are involved in the early course of pediatric UC.

Our finding of increased IL17A expression in UC is in line with recent observations by others. The ratio of mucosal $I L 17 A$ to $I L 17 F$ expression has been shown to correlate significantly with endoscopic disease activity in adult UC. ${ }^{18}$ In addition, increased dual expression of IL17A by $\mathrm{CD} 4^{+} \mathrm{CD} 25^{-}$regulatory $\mathrm{T}$ cells expressing surface transforming growth factor- $\beta$ in its latent form $\left(\mathrm{LAP}^{+}\right)$reduces the suppressor activity of these cells in UC. ${ }^{19}$

A number of groups have examined the ability of measures of immune response type to discriminate CD from UC in adults, but none have made distinctions between CD anatomic subphenotypes or studied newly diagnosed treatment-naive patients exclusively. ${ }^{12,20,21}$ A crosssectional study of adult patients from Japan similarly 


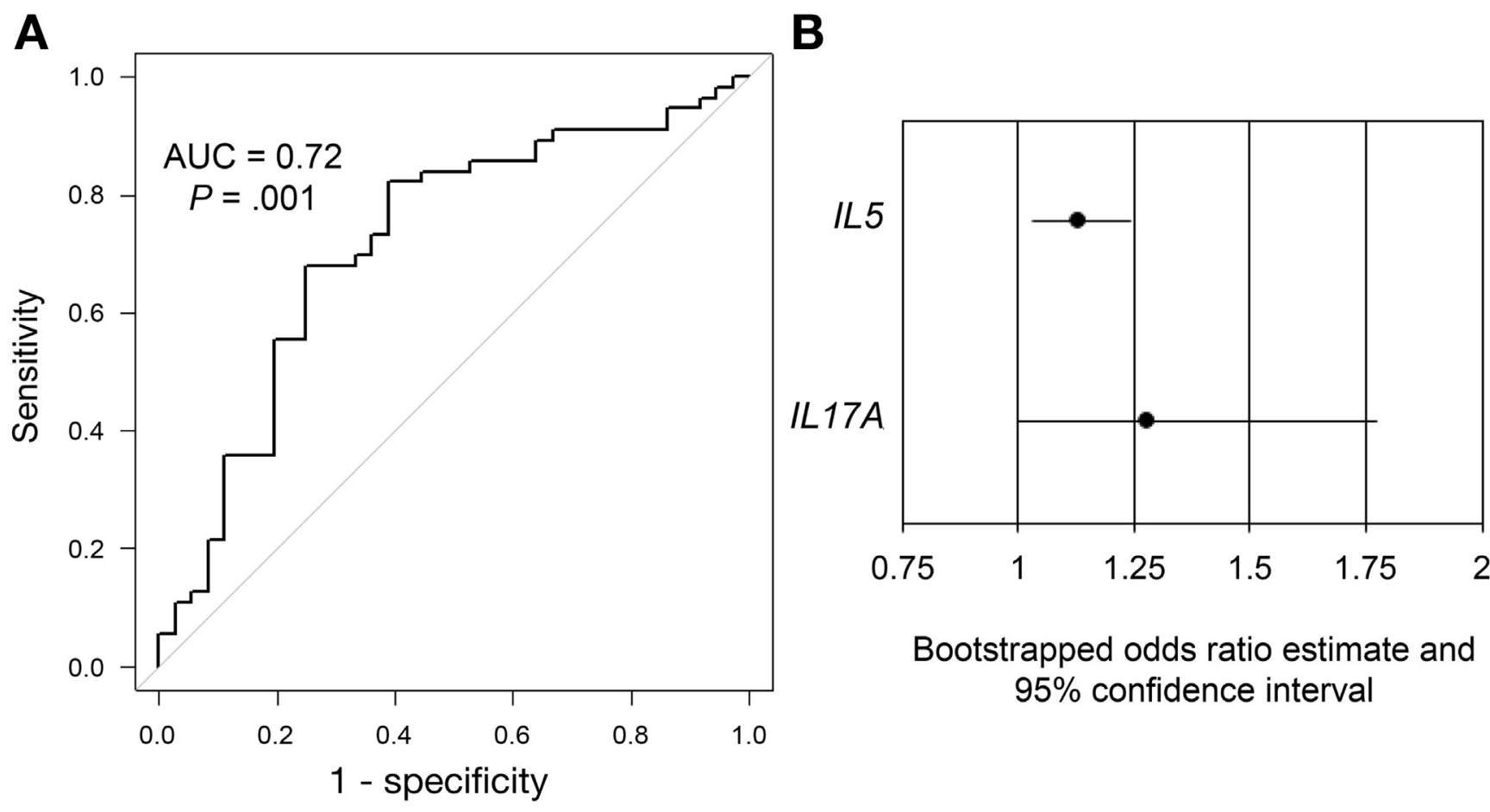

Figure 3. Logistic regression model including IL5 and IL17A gene expression distinguishing UC from CDc. (A) Receiver operator curve of logistic regression model. $(B)$ Chart showing estimates of the ORs (odds of UC per unit increase in Cq) and 95\% Cls for IL5 and IL17A after bootstrap random sample with replacement internal validation. AUC, area under the curve.

showed increased mucosal IL13 gene expression in UC compared with Crohn's disease, and that a panel of genes associated with adaptive immune responses could distinguish the two. ${ }^{12}$ Similarly, another group showed that the higher mucosal type 2 and lower type 1 T cells as measured by flow cytometry distinguishes UC from $\mathrm{CD}$ in adult patients. ${ }^{20}$ By using a discovery cohort of established patients, they showed that a model including the percentage of $\mathrm{CD}^{+}{ }^{+} \mathrm{T}$ cells positive for interferon- $\gamma$, T-bet, IL13, and Gata3 was predictive of $\mathrm{CD}$ over $\mathrm{UC}$, and validated the model in a small cohort of newly diagnosed patients. However, in the former study, tissues samples were taken from both the colon and the ileum, and in both studies the patients had a mean disease duration of at least 8 years, were on a variety of immune-suppressive treatments, and the CD groups included patients with ileitis, ileocolitis, and colitis. Our study substantially builds on these prior findings by showing in a large cohort of newly diagnosed treatmentnaive pediatric patients that rectal type 2 and type 17 gene expression not only distinguishes UC from CD, but distinguishes UC from colon-only CD. By studying tissues from newly diagnosed pediatric patients, our findings provide insight regarding the mucosal immune response from arguably the earliest practical opportunity in the disease course to study IBD. Because patients are treatment naive, we are assured that the findings are not influenced by medications with profound effects on immune function. Furthermore, because colitis-only $\mathrm{CD}$ is more common in children than in adults and can be difficult to distinguish from UC, our study addresses an important clinical problem.
Some investigators have proposed that Crohn's colitis should be considered a distinct disease entity from ileal CD. A recent genome-wide association study meta-analysis showed that based on the relative genetic risk for $\mathrm{CD}$ vs UC, CDc and CDic are best characterized as intermediate phenotypes between UC and CD ileitis (with CDc being intermediate between UC and CDic). ${ }^{22}$ In our immune gene expression panel, IL17A was expressed intermediately in CDc and was significantly different from expression in UC and CDic. CCL11 also showed significantly increased expression in CDc compared with CDic, similar to in UC. Our principal component analysis, which incorporates the collective differences from all the genes analyzed, aggregates CDc patients closer to UC patients, supporting the notion of CDc representing an intermediate phenotype based on gene expression as well as genetic make-up.

Contrary to our initial hypothesis, we observed that pediatric UC patients with a gene expression pattern marked by increased IL13 expression achieved higher rates of steroid-free clinical response and remission, and that IL13 gene expression alone was associated directly with clinical response at 6 and 12 months. The newly diagnosed patients in this study received standard-of-care treatment at the discretion of the treating physician. Other groups have examined markers of adaptive immune responses primarily with regard to response to anti-TNF therapy in adult UC. In a cohort of adults patients with UC, higher mucosal IL17A and IFNG gene expression was associated with remission after infliximab induction therapy. ${ }^{23}$ IL13 expression was not assessed in that study. With regard to markers of type 2 immune responses, one group observed fewer Gata3 ${ }^{+}$ 
lamina propria T cells in UC patients responding to anti-TNF agents compared with those without response. ${ }^{20}$ In addition, IL13RA2 was among several genes identified in a mucosal genome-wide expression study as associated with nonresponse to infliximab in UC. ${ }^{24}$ We did not observe any significant associations between IL17A, IFNG, or IL13RA2 and clinical outcomes in this pediatric UC cohort. The discrepancies between our results and those of other groups likely is owing to our patients being assessed before any IBD-directed therapy, with many achieving remission on either mesalamine or immunomodulator drugs. Only one third of the UC patients in our group were ever exposed to an anti-TNF drug, whereas the patients in these studies were refractory to first-line therapies with response specifically to anti-TNF drugs being assessed.

There are 2 potential explanations as to why increased mucosal IL13 expression at baseline predicts improved clinical outcomes. Investigations by our group and others have supported a pathogenic role for IL13 and type $2 \mathrm{im}$ mune responses in human UC and several murine models of colitis including oxazolone-induced colitis and spontaneous colitis in Wiskott-Aldrich syndrome protein-deficient and T-cell-receptor $\alpha$-deficient mice. ${ }^{2,4,15,25-31}$ If IL13 indeed is part of a pathogenic type 2 immune response, then our results suggest this pathway is suppressed sufficiently by standard initial therapy, and that a subset of patients with increased IL13 expression may be more responsive to treatment. Indeed, we observed that mucosal expression of IL13 was decreased significantly in patients with therapyinduced mucosal healing compared with those with active disease.

However, the failure of 2 phase IIa clinical trials of antiIL13 monoclonal antibodies for the treatment of UC to meet their primary end points draws into question the notion of a pathogenic role of IL13 in UC (although 1 study did meet significance for important secondary end points including clinical remission). ${ }^{32,33}$ It also is possible that improved outcomes in high IL13 expressers is the result of a protective effect exerted by IL13 induced in the context of inflammation. Among UC patients in this study, we did not observe a correlation between IL13 and IL17A expression, suggesting that the production of IL13 may be independent of a Th17 immune response. Indeed, some groups have observed beneficial roles for IL13 with regard to epithelial wound healing and goblet cell function (the latter particularly with regard to helminth expulsion). ${ }^{34-36}$ IL10deficient mice also deficient for IL13RA2, the gene for a neutralizing receptor for IL13, show decreased inflammation when challenged with a parasite or a nonsteroidal antiinflammatory drug, suggesting IL13 activity is protective in these models. ${ }^{37}$ Finally, IL33-dependent group 2 innate lymphoid cells that produce IL5, IL13, and the epidermal growth factor ligand amphiregulin limit inflammation induced from epithelial damage in dextran sodium sulfateinduced colitis in mice. ${ }^{38}$ In line with this last concept, we observed increased expression of the transcript for membrane-bound IL33 receptor, one marker of group 2 innate lymphoid cells, in UC compared with both CD and non-IBD. 
There were several notable strengths to our study. First, our findings were from newly diagnosed treatment-naive pediatric patients, and thus were not influenced by treatment. Second, the cohort was characterized meticulously, allowing us to examine distinct $\mathrm{CD}$ anatomic subphenotypes. Third, the differences we observed were derived from examination of exclusively rectal samples, all from patients with documented rectal involvement. Fourth, we validated gene expression differences between colitis diagnoses in an independent local cohort. A weakness of this study was that only gene expression and not protein abundance was assessed. Tissue samples from the RISK cohort were not collected in a manner conducive to protein analysis. Although cytokines and other proteins may be regulated at the translational or post-translational level, cytokine gene expression measured by real-time RT-qPCR generally correlates quite well with measures of protein abundance. ${ }^{39-41}$ In addition, although we did not observe differences in the expression of the type 1 cytokine $I F N G$ or transcription factor TBX21 between UC and $\mathrm{CD}$, we did not measure expression of other type 1 cytokines such as IL12 or TNFB, thus limiting the conclusions we could draw regarding the relative contribution of a type 1 immune response to UC and CD.

In conclusion, our data support a role for mucosal type 2 inflammatory responses in the early course of pediatric UC. In treatment-naive pediatric patients, UC is distinguished from Crohn's colitis, and specifically colon-only CD by increased expression of genes associated with type 2 and type 17 immune responses. Furthermore, an immune gene expression profile marked by increased expression of the type 2 cytokine $I L 13$ is associated with improved clinical outcomes in pediatric UC. Future studies are warranted from large UC cohorts to determine whether a type 2 gene expression predicts response to specific UC therapies with the ultimate goal of directing therapies based on patient immunophenotype.

\section{Supplementary Material}

Note: To access the supplementary material accompanying this article, visit the online version of Gastroenterology at www.gastrojournal.org, and at http://dx.doi.org/10.1053/ j.gastro.2017.01.016.

\section{References}

1. Fuss IJ, Neurath M, Boirivant $M$, et al. Disparate CD4+ lamina propria (LP) lymphokine secretion profiles in inflammatory bowel disease. Crohn's disease LP cells manifest increased secretion of IFN-gamma, whereas ulcerative colitis LP cells manifest increased secretion of IL-5. J Immunol 1996;157:1261-1270.

2. Fuss IJ, Heller F, Boirivant $M$, et al. Nonclassical CD1d-restricted NK T cells that produce IL-13 characterize an atypical Th2 response in ulcerative colitis. J Clin Invest 2004;113:1490-1497.

3. Wynn TA. Type 2 cytokines: mechanisms and therapeutic strategies. Nat Rev Immunol 2015;15:271-282.
4. Heller F, Florian P, Bojarski C, et al. Interleukin-13 is the key effector Th2 cytokine in ulcerative colitis that affects epithelial tight junctions, apoptosis, and cell restitution. Gastroenterology 2005;129:550-564.

5. Kappelman MD, Moore KR, Allen JK, et al. Recent trends in the prevalence of Crohn's disease and ulcerative colitis in a commercially insured US population. Dig Dis Sci 2012;58:519-525.

6. Adamiak T, Walkiewicz-Jedrzejczak D, Fish D, et al. Incidence, clinical characteristics, and natural history of pediatric IBD in Wisconsin. Inflamm Bowel Dis 2013; 19:1218-1223.

7. Winter DA, Karolewska-Bochenek K, LazowskaPrzeorek I, et al. Pediatric IBD-unclassified is less common than previously reported; results of an 8-year audit of the EUROKIDS registry. Inflamm Bowel Dis 2015;21:2145-2153.

8. Gupta N, Bostrom AG, Kirschner BS, et al. Presentation and disease course in early- compared to later-onset pediatric Crohn's disease. Am J Gastroenterol 2008; 103:2092-2098.

9. de Bie Cl, Pærregaard A, Kolacek S, et al. Disease phenotype at diagnosis in pediatric Crohn's disease. Inflamm Bowel Dis 2013;19:378-385.

10. Levine A, Koletzko S, Turner D, et al. The ESPGHAN revised Porto criteria for the diagnosis of inflammatory bowel disease in children and adolescents. J Pediatr Gastroenterol Nutr 2014;58:795-806.

11. Mosli MH, Feagan BG, Zou G, et al. Development and validation of a histological index for UC. Gut 2015; 66:50-58.

12. Iboshi $Y$, Nakamura K, Ihara E, et al. Multigene analysis unveils distinctive expression profiles of helper T-cellrelated genes in the intestinal mucosa that discriminate between ulcerative colitis and Crohn's disease. Inflamm Bowel Dis 2014;20:967-977.

13. Bliss R, Weinberg J. Determining the probability distribution and evaluating sensitivity and false positive rate of a confounder detection method applied to logistic regression. J Biomet Biostat 2012;3:142.

14. Levine A, Griffiths A, Markowitz J, et al. Pediatric modification of the Montreal classification for inflammatory bowel disease: the Paris classification. Inflamm Bowel Dis 2011;17:1314-1321.

15. Rosen MJ, Frey MR, Washington MK, et al. STAT6 activation in ulcerative colitis: a new target for prevention of IL-13-induced colon epithelial cell dysfunction. Inflamm Bowel Dis 2011;17:2224-2234.

16. Biancheri P, Di Sabatino A, Ammoscato F, et al. Absence of a role for interleukin-13 in inflammatory bowel disease. Eur J Immunol 2014;44:370-385.

17. Kadivar K, Ruchelli ED, Markowitz JE, et al. Intestinal interleukin-13 in pediatric inflammatory bowel disease patients. Inflamm Bowel Dis 2004;10:593-598.

18. Iboshi $\mathrm{Y}$, Nakamura K, Fukaura $\mathrm{K}$, et al. Increased IL-17A/IL-17F expression ratio represents the key mucosal $\mathrm{T}$ helper/regulatory cell-related gene signature paralleling disease activity in ulcerative colitis. J Gastroenterol 2017;52:315-326. 
19. D’Ambrosio A, Cossu A, Amendola A, et al. Lamina propria CD4+LAP+ regulatory $T$ cells are increased in active ulcerative colitis but show increased IL-17 expression and reduced suppressor activity. J Crohns Colitis 2016;10:346-353.

20. Li J, Ueno A, Fort Gasia M, et al. Profiles of lamina propria $T$ helper cell subsets discriminate between ulcerative colitis and Crohn's disease. Inflamm Bowel Dis 2016;22:1779-1792.

21. Verdier J, Begue B, Cerf-Bensussan N, et al. Compartmentalized expression of Th1 and Th17 cytokines in pediatric inflammatory bowel diseases. Inflamm Bowel Dis 2012;18:1260-1266.

22. Cleynen I, Boucher G, Jostins L, et al. Inherited determinants of Crohn's disease and ulcerative colitis phenotypes: a genetic association study. Lancet 2016; 387:156-167.

23. Rismo R, Olsen T, Cui G, et al. Mucosal cytokine gene expression profiles as biomarkers of response to infliximab in ulcerative colitis. Scand J Gastroenterol 2012; 47:538-547.

24. Arijs I, Li K, Toedter G, et al. Mucosal gene signatures to predict response to infliximab in patients with ulcerative colitis. Gut 2009;58:1612-1619.

25. Heller F, Fuss IJ, Nieuwenhuis EE, et al. Oxazolone colitis, a Th2 colitis model resembling ulcerative colitis, is mediated by IL-13-producing NK-T cells. Immunity 2002; 17:629-638.

26. Mannon PJ, Hornung RL, Yang Z, et al. Suppression of inflammation in ulcerative colitis by interferon- $\beta-1 \mathrm{a}$ is accompanied by inhibition of IL-13 production. Gut 2011; 60:449-455.

27. Fuss IJ, Joshi B, Yang Z, et al. IL-13R 2-bearing, type II NKT cells reactive to sulfatide self-antigen populate the mucosa of ulcerative colitis. Gut 2014;63: 1728-1736.

28. Rosen MJ, Chaturvedi R, Washington MK, et al. STAT6 deficiency ameliorates severity of oxazolone colitis by decreasing expression of claudin-2 and Th2-inducing cytokines. J Immunol 2013;190:1849-1858.

29. Mizoguchi A, Mizoguchi E, Bhan AK. The critical role of interleukin 4 but not interferon gamma in the pathogenesis of colitis in T-cell receptor alpha mutant mice. Gastroenterology 1999;116:320-326.

30. Nguyen DD, Maillard MH, Cotta-de-Almeida V, et al. Lymphocyte-dependent and Th2 cytokine-associated colitis in mice deficient in Wiskott-Aldrich syndrome protein. Gastroenterology 2007;133:1188-1197.

31. Kawashima R, Kawamura YI, Oshio T, et al. Interleukin13 damages intestinal mucosa via TWEAK and Fn14 in mice-a pathway associated with ulcerative colitis. Gastroenterology 2011;141:2119-2129.

32. Danese S, Rudziński J, Brandt W, et al. Tralokinumab for moderate-to-severe UC: a randomised, double-blind, placebo-controlled, phase Ila study. Gut 2015;64: 243-249.

33. Reinisch W, Panés J, Khurana S, et al. Anrukinzumab, an anti-interleukin 13 monoclonal antibody, in active UC: efficacy and safety from a phase lla randomised multicentre study. Gut 2015;64:894-900.
34. Seno H, Miyoshi H, Brown SL, et al. Efficient colonic mucosal wound repair requires Trem2 signaling. Proc Natl Acad Sci U S A 2009;106:256-261.

35. Webb RA, Hoque $T$, Dimas $S$. Expulsion of the gastrointestinal cestode, Hymenolepis diminuta by tolerant rats: evidence for mediation by a Th2 type immune enhanced goblet cell hyperplasia, increased mucin production and secretion. Parasite Immunol 2007;29:11-21.

36. McKay DM, Khan WI. STAT-6 is an absolute requirement for murine rejection of Hymenolepis diminuta. J Parasitol 2003;89:188-189.

37. Wilson MS, Ramalingam TR, Rivollier A, et al. Colitis and intestinal inflammation in IL10-/- mice results from IL-13R $\alpha 2$-mediated attenuation of IL-13 activity. Gastroenterology 2011;140:254-264.

38. Monticelli LA, Osborne LC, Noti M, et al. IL-33 promotes an innate immune pathway of intestinal tissue protection dependent on amphiregulin-EGFR interactions. Proc Natl Acad Sci U S A 2015;112:10762-10767.

39. Young S-H, Antonini JM, Roberts JR, et al. Performance evaluation of cytometric bead assays for the measurement of lung cytokines in two rodent models. J Immunol Methods 2008;331:59-68.

40. Sullivan KE, Cutilli J, Piliero LM, et al. Measurement of cytokine secretion, intracellular protein expression, and mRNA in resting and stimulated peripheral blood mononuclear cells. Clin Diagn Lab Immunol 2000;7:920-924.

41. Flores MG, Zhang S, Ha A, et al. In vitro evaluation of the effects of candidate immunosuppressive drugs: flow cytometry and quantitative real-time PCR as two independent and correlated read-outs. J Immunol Methods 2004;289:123-135.

Received September 22, 2016. Accepted January 23, 2017.

\section{Reprint requests}

Address requests for reprints to: Michael J. Rosen, MD, MSCl, Division of Gastroenterology, Hepatology and Nutrition, Cincinnati Children's Hospital Medical Center, MLC 2010, Cincinnati, Ohio 45229. e-mail: michael.rosen@cchmc.org; fax: (513) 636-5581.

\section{Acknowledgments}

The authors thank the following RISK study investigators for their efforts in enrolling, phenotyping, and following up participants in this cohort: Anthony R. Otley, Scott B. Snapper, Stephen L. Guthery, David J. Keljo, Barbara S. Kirschner, Marian D. Pfefferkorn, Maria Oliva-Hemker, Ashish S. Patel, Shervin Rabizadeh, Stanley A. Cohen, David A. Ziring, and Jonathan Evans. Neal S. Leleiko enrolled research participants and secured biospecimens, and critical revision of the manuscript for important intellectual content. The authors also thank the patients and their families who participated in this study.

\section{Conflicts of interest}

These authors disclose the following: Michael J. Rosen has served on an advisory board for Abbvie and receives research support from Prometheus Laboratories; Jeffrey S. Hyams has served on advisory boards for Janssen and Abbvie, and as a consultant for Lilly, Celgene, Takeda, Astra Zeneca, and Receptos; David R. Mack has served on advisory boards for Abbvie, Janssen, and Mead Johnson, as a consultant for UCB, and is a shareholder of Biotagenics; Melvin B. Heyman has received research support from Janssen, Genentech, Sucampo, and Abbvie, and has served as an advisor for Gilead; Michael D. Kappelman has received research support from and served as a consultant for Janssen and Abbvie; Joel R. Rosh has received research support from and served as an advisor for Janssen and Abbvie; Thomas D. Walters has received speaker fees and research support from and served on advisory boards for Janssen Canada and Abbvie Canada; and Lee $A$. Denson has received research support from Janssen and royalties from Glycosyn, LLC. The remaining authors disclose no conflicts. 
Funding

Research reported in this publication was supported primarily by the National Institute of Diabetes and Digestive and Kidney Diseases of the National Institutes of Health K23DK094832 (M.J.R.). Biospecimens were obtained from the Risk Stratification and Identification of Immunogenetic and Microbial Markers of Rapid Disease Progression in Children with Crohn's Disease (RISK) Study, funded by the Crohn's and Colitis Foundation. This project also was supported in part by the National Institutes of Health Clinical and Translational Science Award 1UL1TR001425-01 (Research Electronic Data Capture (REDCap)
Database), National Institute of Diabetes and Digestive and Kidney Diseases P30DK078392 (Gene and Protein Expression Core) of the Digestive Disease Research Core Center in Cincinnati, National Institutes of Health P30ES006096 of the University of Cincinnati College of Medicine Center for Environmental Genetics (Genomics, Epigenomics, and Sequencing Core), National Institutes of Health R01DK090119 and the Crohn's and Colitis Foundation Senior Research Award (S.P.H.), Nationa Institutes of Health K23DK105229 (P.M.), and the Gutsy Kids Fund including a philanthropic donation from the Karen and Brock Wagner Family (R.K.). 
A

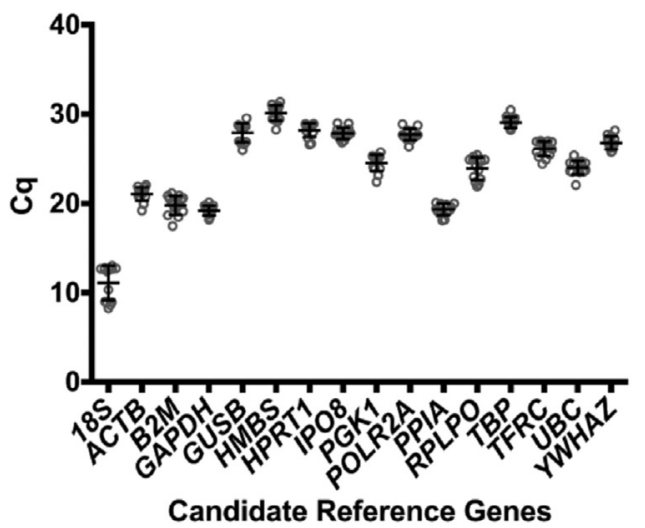

B

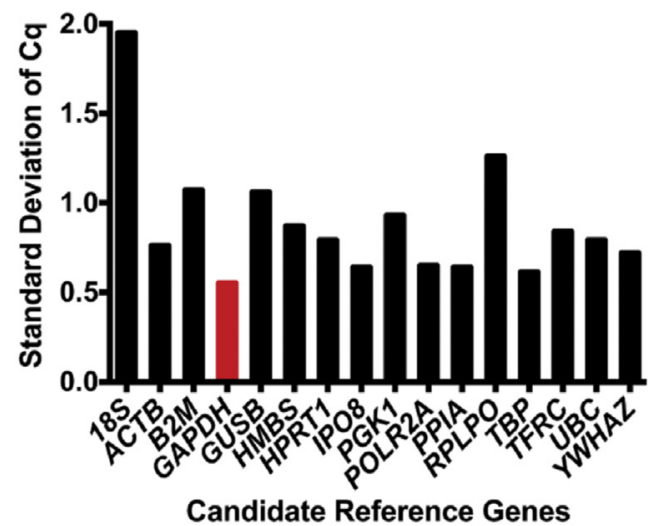

Supplementary Figure 1. Identification of glyceraldehyde-3-phosphate dehydrogenase (GAPDH) as the reference gene with the least expression variation across diagnosis groups. (A) Dot plot showing the mean Cq and standard errors of 16 candidate reference genes from an endogenous control real-time RT-qPCR microfluidic array $(\mathrm{n}=4$ each of non-IBD, CDic, CDc, and UC). (B) Bar chart showing SDs of the $\mathrm{Cq}$ for each candidate reference gene. GAPDH showed the lowest variability in expression across samples. 
A

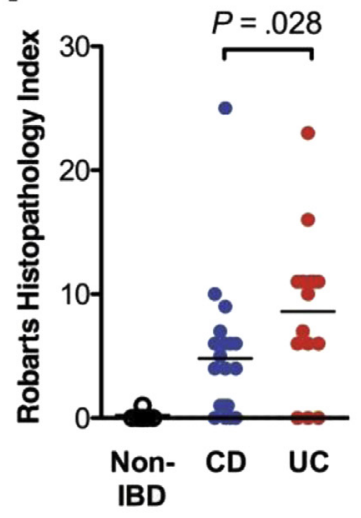

C

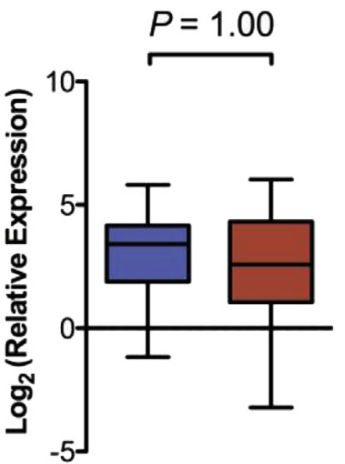

IFNG

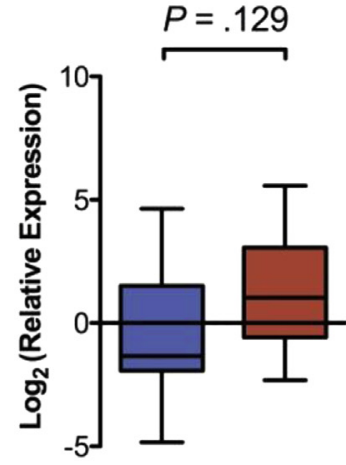

IL5
B
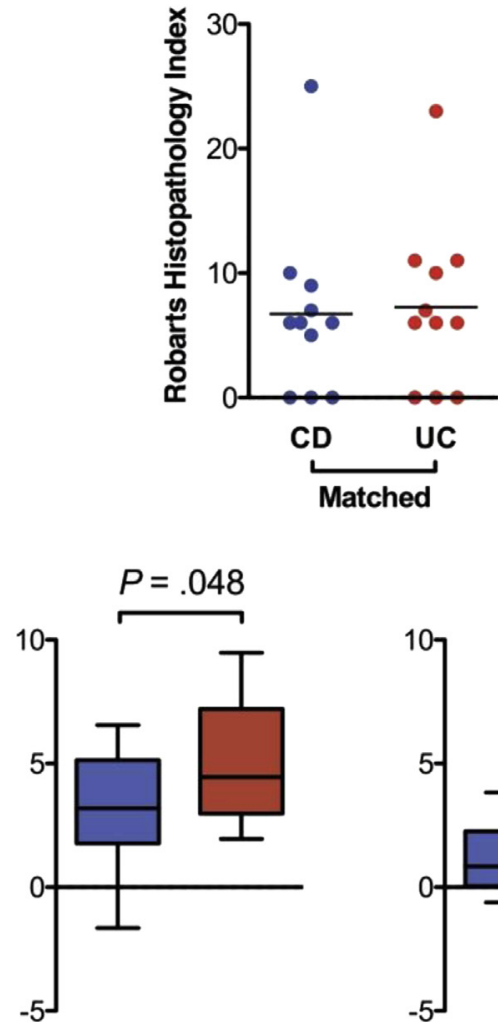

IL13

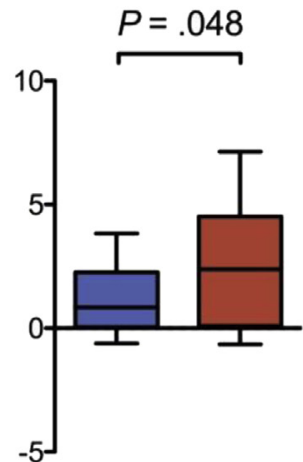

\section{IL13RA2}

$P=.160$

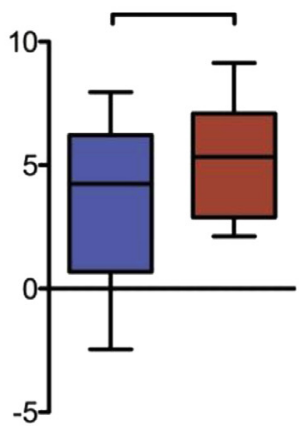

IL17A

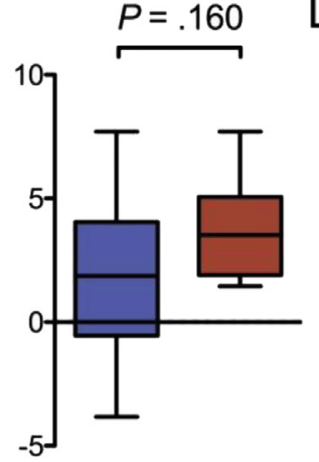

IL23A

Supplementary Figure 2. Analysis of Cincinnati cohort rectal mucosa real-time RT-qPCR controlling for histologic disease activity. (A) Dot plot of RHI scores for CD and UC patients in the Cincinnati cohort. (B) Dot plot of a subset of CD and UC patients in the Cincinnati cohort matched on RHI scores. (C) Box and whisker chart showing gene expression normalized to median expression of the non-IBD patient group in CD and UC patients matched on RHI scores (boxes represent medians and interquartile range, whiskers represent the $95 \% \mathrm{Cl}$ ). 
Supplementary Table 1.Targets on Gene Expression Array

Gene

IL4

IL5

IL13

IL13RA2

IL33

IL1RL1(s)

IL1RL1(m)

CCL11

CHI3L1

ICOS

GATA3

RORA

CLDN2

IFNG

TBX21

IL17A

IL23A

RORC

IL22

AHR

IL10

TGFB1

S100A8

GAPDH
Protein function

Assay ID

Type 2 cytokine

Type 2 cytokine

Type 2 cytokine

Type 2 cytokine receptor/protein induced by IL13

Cytokine that induces type 2 immune responses

IL33 receptor (soluble) that regulates type 2 immune responses

IL33 receptor (membrane) that induces type 2 immune responses

Chemokine associated with eosinophil recruitment and type 2 immune responses

Protein induced by type 2 immune responses

T-cell co-stimulatory molecular that augments type 2 immune responses

Type 2 T-cell transcription factor

Group 2 innate lymphoid cell transcription factor

Tight junction protein induced by IL13

Type 2 cytokine

Type 1 transcription factor

Type 17 cytokine

Cytokine that augments type 17 immune responses

Type 17 transcription factor

Type 17 cytokine

Group 3 ILC transcription factor

T-regulatory cell cytokine

Cytokine that induces T-regulatory cell and type $17 \mathrm{~T}$-cell differentiation

Component of calprotectin/marker of inflammation

Reference gene
Hs00174122_m1

Hs00174200_m1

Hs00174379_m1

Hs00152924 m1

Hs01125943_m1

Hs01073297_m1

Hs01073295_m1

Hs00237013_m1

Hs00609691_m1

Hs00359999_m1

Hs00231122_m1

$\mathrm{Hs} 00536545 \mathrm{~m} 1$

Hs01549234_m1

Hs00174143_m1

Hs00203436_m1

Hs00174383_m1

Hs00372324_m1

Hs01076122_m1

Hs01574154_m1

Hs00169233_m1

Hs00174086_m1

Hs00171257_m1

Hs00374264_g1

Hs99999905_m1 
Supplementary Table 2. Comparison Between the Studied RISK Cohort Subset and Overall RISK Cohort

$$
\text { Non-IBD CDic CDc }
$$

\begin{tabular}{|c|c|c|c|c|c|c|c|c|c|}
\hline & $(N=1812)$ & $(\mathrm{N}=49)$ & $(N=408)$ & $(N=46)$ & $(N=606)$ & $(\mathrm{N}=36)$ & $(\mathrm{N}=210)$ & $(N=56)$ & $(N=200)$ \\
\hline Age, $y$ & $12.7(9.9,14.9)$ & $12.8(10.8,15.0)$ & $12.7(9.5,15.0)$ & $12.4(10.9,13.6)$ & $12.4(9.9,14.7)$ & $12.7(10.8,14.5)$ & $12.5(9.4,15.0)$ & $13.5(10.8,15.5)$ & $13.1(10.2,15.0)$ \\
\hline A1a: 0 to $<10 y$ & $460(25.4)$ & $9(18.4)$ & $116(28.7)$ & $11(23.9)$ & $152(25.1)$ & $6(17.1)$ & $57(27.1)$ & $13(23.2)$ & $49(24.5)$ \\
\hline A1b: 10 to $<17$ y & $1348(74.6)$ & 39 (79.6) & 288 (71.3) & 35 (76.1) & 454 (74.9) & $30(85.7)$ & $153(72.9)$ & $43(76.8)$ & $151(75.5)$ \\
\hline Male sex & 1055 (58.2) & $23(46.9)$ & $226(55.4)$ & $25(54.3)$ & 377 (62.2) & 17 (47.2) & $120(57.1)$ & $30(53.6)$ & $106(53.0)$ \\
\hline \multicolumn{10}{|l|}{ Diagnosis } \\
\hline Non-IBD & $408(22.5)$ & 49 (100) & 408 (100) & $0(0)$ & $0(0)$ & $0(0)$ & $0(0)$ & $0(0)$ & $0(0)$ \\
\hline$C D$ & $1118(61.7)$ & $0(0)$ & $0(0)$ & 46 (100) & 606 (100) & 36 (100) & $210(100)$ & $0(0)$ & $0(0)$ \\
\hline UC & $200(11.0)$ & $0(0)$ & $0(0)$ & $0(0)$ & $0(0)$ & $0(0)$ & $0(0)$ & $56(100)$ & $200(100)$ \\
\hline IBD-U & $86(4.7)$ & $0(0)$ & $0(0)$ & $0(0)$ & $0(0)$ & $0(0)$ & $0(0)$ & $0(0)$ & $0(0)$ \\
\hline \multicolumn{10}{|l|}{ CD location ${ }^{a}$} \\
\hline L1 & $181(16.2)$ & & & $0(0)$ & $0(0)$ & $0(0)$ & $0(0)$ & & \\
\hline L2: & 210 (18.8) & & & $0(0)$ & $0(0)$ & 36 (100) & $210(100)$ & & \\
\hline L3 & 606 (54.2) & & & $46(100)$ & $606(100)$ & $0(0)$ & $0(0)$ & & \\
\hline L4 only & $18(1.6)$ & & & $0(0)$ & $0(0)$ & $0(0)$ & $0(0)$ & & \\
\hline Insufficient data & $103(9.2)$ & & & $0(0)$ & $0(0)$ & $0(0)$ & $0(0)$ & & \\
\hline \multicolumn{10}{|l|}{ Upper GI } \\
\hline L4a & $531(47.5)$ & & & 32 (69.6) & $322(53.1)$ & 17 (47.2) & $82(39.0)$ & & \\
\hline $\mathrm{L} 4 \mathrm{~b}$ & $147(13.1)$ & & & $12(26.1)$ & 97 (16.0) & $0(0)$ & $20(9.5)$ & & \\
\hline \multicolumn{10}{|l|}{ UC extent ${ }^{b}$} \\
\hline E1 & $5(2.5)$ & & & & & & & $2(3.6)$ & $5(2.5)$ \\
\hline E2 & $26(13.0)$ & & & & & & & 7 (12.5) & $26(13.0)$ \\
\hline E3 & $22(11.0)$ & & & & & & & 7 (12.5) & $22(11.0)$ \\
\hline E4 & $112(56.0)$ & & & & & & & $36(64.3)$ & $112(56.0)$ \\
\hline Insufficient data & 35 (17.5) & & & & & & & $4(7.1)$ & 35 (17.5) \\
\hline $\begin{array}{l}\text { Macroscopic rectal } \\
\text { involvement }\end{array}$ & $846(60.3)$ & & & $46(100)$ & 409 (67.5) & $36(100)$ & $157(74.8)$ & 56 (100) & $177(88.5)$ \\
\hline \multicolumn{10}{|l|}{ PGA } \\
\hline Inactive & $50(3.6)$ & & & $0(0)$ & $13(2.1)$ & $1(2.8)$ & $11(5.2)$ & $1(1.8)$ & $7(3.5)$ \\
\hline Mild & $458(32.6)$ & & & $11(23.9)$ & $168(27.7)$ & 7 (19.4) & $62(29.5)$ & $20(35.7)$ & $63(31.5)$ \\
\hline Moderate & $684(48.7)$ & & & $22(47.8)$ & $324(53.5)$ & $19(52.8)$ & $106(50.5)$ & $23(41.1)$ & $101(50.5)$ \\
\hline Severe & 209 (14.9) & & & $13(28.3)$ & 98 (16.2) & $9(25.0)$ & 31 (14.8) & $12(21.4)$ & $29(14.5)$ \\
\hline Insufficient data & $3(0.2)$ & & & $0(0)$ & $3(0.5)$ & $0(0)$ & $0(0)$ & $0(0)$ & $0(0)$ \\
\hline PUCAI & $45(30,60)$ & & & & & & & $45(35,60)$ & $45(30,60)$ \\
\hline Rectal deep ulcers & $158(11.3)$ & & & $5(10.9)$ & 75 (12.4) & $12(33.3)$ & $36(17.1)$ & $10(17.9)$ & $28(14.0)$ \\
\hline
\end{tabular}

UC

Entire RISK cohort Studied subset RISK cohort Studied subset RISK cohort Studied subset RISK cohort Studied subset RISK cohort

NOTE. Quantitative variables are expressed as medians (quartile 1, quartile 3), and dichotomous variables are shown as $\mathrm{n}$ (\%).

IBD-U, inflammatory bowel disease unclassified; PUCAI, Pediatric Ulcerative Colitis Activity Index.

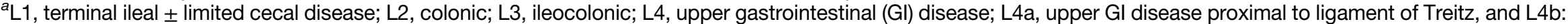
upper Gl disease distal to ligament of Treitz.

${ }^{b} \mathrm{E} 1$, ulcerative proctitis; E2, left-sided colitis; E3, extensive colitis; E4, pancolitis. 
Supplementary Table 3.Characteristics of the Cincinnati Cohort Patients

\begin{tabular}{|c|c|c|c|}
\hline & Non-IBD $(n=17)$ & $C D(n=20)$ & $U C(n=14)$ \\
\hline Age at biopsy collection, $y$ & $15.8(10.5,16.6)$ & $15.0(11.2,6.7)$ & $18.0(14.5,19.0)$ \\
\hline Male sex & 7 (41.2) & $15(75.0)$ & $25(54.3)$ \\
\hline Age at diagnosis, $y$ & & $10.6(9.2,10.6)$ & $14.4(11.6,16.5)$ \\
\hline A1a: 0 to $<10$ y & & $8(40.0)$ & $3(21.4)$ \\
\hline A1b: 10 to $<17$ y & & $11(55.0)$ & $10(71.4)$ \\
\hline A2: $17-40$ y & & $1(5.0)$ & $1(7.1)$ \\
\hline Time since diagnosis, $y$ & & $2.2(0,4.7)$ & $2.8(1.0,6.2)$ \\
\hline Biopsy specimen collected at diagnostic endoscopy & & $9(45.0)$ & $0(0.0)$ \\
\hline \multicolumn{4}{|l|}{ CD location } \\
\hline L1: terminal ileal \pm limited cecal disease & & $0(0)$ & $0(0)$ \\
\hline L2: colonic & & $8(40.0)$ & $0(0)$ \\
\hline L3: ileocolonic & & $12(60.0)$ & $46(100)$ \\
\hline L4a: upper disease proximal to ligament of Treitz & & $11(55.0)$ & $32(69.6)$ \\
\hline L4b: upper disease distal to ligament of Treitz & & $2(10.0)$ & $12(26.1)$ \\
\hline \multicolumn{4}{|l|}{ UC extent } \\
\hline E1: ulcerative proctitis & & & $1(7.1)$ \\
\hline E2: left-sided colitis & & & $3(21.4)$ \\
\hline E3: extensive colitis & & & $3(21.4)$ \\
\hline E4: pancolitis & & & $7(50.0)$ \\
\hline Macroscopic rectal involvement & & $20(100)$ & $14(100)$ \\
\hline \multicolumn{4}{|l|}{ Clinical disease activity } \\
\hline shPCDAl & & $25(12.5,35)$ & \\
\hline PUCAI & & & $30(15,60)$ \\
\hline Endoscopic disease activity & & & $22(47.8)$ \\
\hline SES-CD & & $12(8.5,17)$ & \\
\hline \multicolumn{4}{|l|}{ Mayo endoscopic score } \\
\hline Mild & & & $4(28.6)$ \\
\hline Moderate & & & $3(21.4)$ \\
\hline Severe & & & $7(50.0)$ \\
\hline \multicolumn{4}{|l|}{ Medications } \\
\hline None & & $5(25.0)$ & $0(0.0)$ \\
\hline Oral corticosteroids & & $2(10.0)$ & $7(50.0)$ \\
\hline Rectal corticosteroids & & $1(5.0)$ & $2(14.3)$ \\
\hline Oral mesalamine & & $4(20.0)$ & $10(71.4)$ \\
\hline Antibiotic & & $3(15.0)$ & $0(0.0)$ \\
\hline 6-mercaptopurine or azathioprine & & $5(25.0)$ & $2(14.3)$ \\
\hline Methotrexate & & $0(0.0)$ & $1(7.1)$ \\
\hline Anti-TNF biologic & & $2(10.0)$ & $3(21.4)$ \\
\hline
\end{tabular}

NOTE. Quantitative variables are expressed as medians (quartile 1, quartile 3), and dichotomous variables are shown as $n$ (\%). PUCAI, Pediatric Ulcerative Colitis Activity Index; SES-CD, Simple Endoscopic Score for Crohn's Disease; shPCDAI, Short Pediatric Crohn's Disease Activity Index.

Supplementary Table 4. Univariate Logistic Regression for Discriminating UC From CDc

\begin{tabular}{lccc}
\multicolumn{1}{c}{ Gene } & OR $^{a}$ & $95 \% \mathrm{Cl}$ & $P$ value \\
IL5 & 1.147 & $1.050-1.644$ & .003 \\
IL13 & 1.100 & $0.998-1.212$ & .056 \\
IL13RA2 & 1.311 & $1.045-1.644$ & .020 \\
IL1RL1(m) & 1.798 & $1.129-2.865$ & .014 \\
ICOS & 1.435 & $0.923-2.229$ & .109 \\
IL17A & 1.265 & $1.032-1.551$ & .024 \\
IL23A & 1.391 & $1.063-1.821$ & .016
\end{tabular}


Supplementary Table 5. Change in Effect Estimate After Bivariate Analyses With S100A8

Effect estimate

\begin{tabular}{lrrr}
\multicolumn{1}{c}{ Gene } & Univariate & Bivariate & Change, \% \\
IL5 & & & -8.6 \\
IL13 & 0.138 & 0.126 & -18.1 \\
IL13RA2 & 0.095 & 0.078 & +22.8 \\
IL1RL1 $(m)$ & 0.270 & 0.332 & -4.4 \\
ICOS & 0.587 & 0.561 & -23.3 \\
IL17A & 0.361 & 0.277 & -1.7 \\
IL23A & 0.235 & 0.232 & -0.7
\end{tabular}

Supplementary Table 6. Inclusion of S100A8 in Multivariate Logistic Regression Model for Discriminating UC From CDc

$\begin{array}{lccc}\text { Gene } & \text { OR }^{a} & 95 \% \mathrm{Cl} & P \text { value } \\ \text { IL5 } & 1.133 & 1.032-1.238 & .009 \\ \text { IL17A } & 1.232 & 0.976-1.467 & .132 \\ \text { S100A8 } & 0.959 & 0.747-1.233 & .747\end{array}$

${ }^{a}$ Odds of a diagnosis of UC over CDc per unit increase in $\mathrm{Cq}$ value for the listed gene. 
Supplementary Table 7.Univariate Logistic Regression of Gene Expression for Predicting UC Clinical Outcomes

\begin{tabular}{|c|c|c|c|c|c|c|c|c|}
\hline \multirow[b]{3}{*}{ Gene } & \multicolumn{4}{|c|}{ Remission } & \multicolumn{4}{|c|}{ Response } \\
\hline & \multicolumn{2}{|l|}{6 month } & \multicolumn{2}{|l|}{12 month } & \multicolumn{2}{|l|}{6 month } & \multicolumn{2}{|l|}{12 month } \\
\hline & $\mathrm{OR}^{a}(95 \% \mathrm{Cl})$ & $P$ value & OR $(95 \% \mathrm{Cl})$ & $P$ value & OR $(95 \% \mathrm{Cl})$ & $P$ value & OR $(95 \% \mathrm{Cl})$ & $P$ value \\
\hline IL5 & $1.119(0.978-1.279)$ & .104 & $1.050(0.921-1.198)$ & .466 & $1.116(0.981-1.271)$ & .095 & $1.126(0.979-1.294)$ & .094 \\
\hline IL13 & $1.115(0.973-1.279)$ & .118 & $1.126(0.978-1.297)$ & .099 & $1.182(1.028-1.359)$ & .019 & $1.172(1.012-1.359)$ & .034 \\
\hline IL13RA2 & $1.350(0.939-1.942)$ & .105 & $0.847(0.595-1.208)$ & .359 & $1.206(0.855-1.701)$ & .286 & $1.046(0.733-1.493)$ & .804 \\
\hline $\operatorname{IL} 1 R L 1(\mathrm{~m})$ & $1.241(0.618-2.494)$ & .543 & $0.716(0.331-1.550)$ & .397 & $1.751(0.786-3.891)$ & .144 & $0.690(0.314-1.517)$ & .356 \\
\hline ICOS & $1.151(0.581-2.283)$ & .686 & $1.198(0.631-2.273)$ & .582 & $1.032(0.515-2.066)$ & .930 & $1.103(0.570-2.137)$ & .772 \\
\hline IL17A & $1.253(0.919-1.706)$ & .153 & $1.111(0.854-1.445)$ & .433 & 1.239 (0.929-1.650) & .170 & $1.117(0.861-1.449)$ & .407 \\
\hline IL23A & $1.311(0.887-1.934)$ & .175 & $1.071(0.746-1.538)$ & .711 & $1.199(0.813-1.767)$ & .360 & $1.047(0.718-1.527)$ & .810 \\
\hline
\end{tabular}

NOTE. Bold text indicates $P<.05$.

${ }^{a}$ Odds of outcome per unit increase in $\mathrm{Cq}$ value for the listed gene.

Supplementary Table 8. Comparison of Baseline Characteristics and Medication Exposures Between UC Patient Gene Expression Clusters

Clusters 1-3 Clusters 4 and $5 \quad P$

$$
(n=33) \quad(n=13) \quad \text { value }
$$

Baseline characteristics

$\begin{array}{lccc}\text { Age, } y & 13.6(10.7-15.1) & 12.2(11.5-15.8) & .813 \\ \text { Male sex } & 19(57.6) & 5(38.5) & .330 \\ \text { UC extent } & & & \\ \text { E1: ulcerative } & 1(3.1) & 0(0) & .246^{a} \\ \quad \text { proctitis } & & & \\ \text { E2: left-sided colitis } & 2(6.3) & 2(15.4) & \\ \text { E3: extensive colitis } & 4(12.5) & 4(30.8) & \\ \text { E4: pancolitis } & 25(78.1) & 7(53.8) & \\ \text { Data not available } & 1(3.1) & 0(0) & \\ \text { PGA } & & & \\ \text { Inactive } & 1(3.0) & 0(0) & .546^{a} \\ \text { Mild } & 15(45.5) & 4(30.8) & \\ \text { Moderate } & 9(27.3) & 5(38.5) & \\ \text { Severe } & 8(24.2) & 4(30.8) & \\ \text { Rectal deep ulcers } & 5(15.6) & 2(15.4) & 1.00 \\ \text { Medication exposures } & & & \\ \text { 6 months } & \mathrm{n}=31 & \mathrm{n}=13 & \\ \text { Corticosteroids } & 24(77.4) & 12(92.3) & .401 \\ \text { Mesalamine } & 24(77.4) & 11(84.6) & .703 \\ \text { Thiopurines } & 10(32.3) & 6(46.2) & .496 \\ \text { Methotrexate } & 0(0) & 0(0) & - \\ \text { Anti-TNF biologic } & 5(16.1) & 4(30.8) & .414 \\ \text { 12 months } & \mathrm{n}=28 & \mathrm{n}=11 & \\ \text { Corticosteroids } & 23(82.1) & 0(90.9) & .655 \\ \text { Mesalamine } & 22(78.6) & 9(81.8) & 1.00 \\ \text { Thiopurines } & 12(46.4) & 4(36.4) & .725 \\ \text { Methotrexate } & 1(3.6) & 1(9.1) & .489 \\ \text { Anti-TNF biologic } & 7(25.0) & 6(54.5) & .131 \\ & & & \end{array}$

NOTE. Quantitative variables are expressed as medians (quartile 1, quartile 3) and dichotomous variables are shown as $\mathrm{n}(\%)$.

${ }^{a}$ First 2 groups were combined for the chi-square test. 